System bei den betroffenen Leistungen vorsieht, auch dann tragen muss, wenn es sie im Behandlungsstaat nicht gibt, er andererseits aber volle Erstattung der Kosten verlangen kann, wenn Selbstbehalte nur im Behandlungsstaat, nicht aber im Versicherungsstaat existieren. Diese in der Literatur vorherrschende Auffassung ist nicht unbestritten ${ }^{45}$ und sie entspricht, wie eine neuere Entscheidung zeigt, auch nicht der Auffassung des EuGH. Der EuGH ${ }^{46}$ differenziert bei der Höhe der zu erstattenden Kosten danach, ob es sich um eine unvorhergesehene oder eine geplante Behandlung handelt. Nur bei einer geplanten Behandlung könne eine gegenüber den tatsächlichen Kosten geringere Erstattung den Unionsbürger davon abhalten, eine Behandlung im anderen Mitgliedstaat in Anspruch zu nehmen, was eine Behinderung des freien Dienstleistungsverkehrs darstelle! Als Konsequenz müssten demnach gerade die finanziell schwach ausgestatteten Gesundheitssysteme der osteuropäischen Mitgliedstaaten bei Behandlungen in einem westeuropäischen Land die dort maßgebenden relativ hohen Vergütungssätze übernehmen ${ }^{47}$.

\section{Fazit: keine Harmonisierung der Gesundheitssysteme in Europa ohne gemeinsame Sozial- und Wirtschaftsordnung}

Die Entwicklung der grenzüberschreitenden Inanspruchnahme von Gesundheitsleistungen innerhalb der EU ist ein Musterbeispiel für eine allmähliche Erosion nationalstaatlicher Kompetenzen zugunsten zentraler Regelungen im Gemeinschaftsrecht. Die einseitige Ausrichtung der Rechtsprechung des EuGH zur grenzüberschreitenden Inanspruchnahme von Gesundheitsleistungen an den Grundfreiheiten des Unionsvertrages, die ja maßgeblich durch die ursprüngliche Ausrichtung der Union als Wirtschaftsgemeinschaft geprägt sind, kann jedenfalls nicht überzeugen. Sie übersieht, dass eine Harmonisierung der Sozialordnung der Gemeinschaft einer gemeinsamen Wirt- schaftsordnung bedarf und die ökonomische Leistungskraft einen Mindeststandard verlangt, der ausreicht, die Finanzierung einer Gesundheitsversorgung auf einheitlichem Niveau sicherzustellen. Solange dies nicht der Fall ist, wäre es sachgerechter gewesen, die mitgliedstaatliche Ausgestaltung der Gesundheitssysteme zu tolerieren, in denen eine Balance zwischen Dienstleistungsinteressen und optimalem Ressourceneinsatz angestrebt wird. Die ständig wiederkehrende Betonung der Respektierung der nationalstaatlichen Autonomie auf dem Gebiet der Versorgung mit Gesundheitsleistungen in der Rechtsprechung des EuGH erweist sich jedenfalls als inhaltsloses Lippenbekenntnis.

Mit der Patienten-Mobilitäts-RL werden die Ergebnisse dieser Rechtsprechung allen Mitgliedstaaten als Normprogramm vorgegeben und zugleich sollen die nationalen Versicherungsträger und Leistungserbringer für die Möglichkeiten grenzüberschreitender Inanspruchnahme von Gesundheitsleistungen Reklame machen, obgleich der Gerichtshof die Eingriffe in die Sozial- bzw. Gesundheitssysteme der Mitgliedstaaten gerade damit gerechtfertigt hat, dass sich die ökonomischen Auswirkungen wegen der nicht übermäßigen Nachfrage in Grenzen hielten. Eine solche Taktik ist nicht geeignet, das Vertrauen in die Organe der Gemeinschaft zu stärken!

45) Vgl. Eichenhofer, Sozialrecht in der Europäischen Union, 3. Aufl. 2006, \$8, Rdnr. 204, dort weitere Nachweise in Fn. 37.

46) EuGH, Urt. v. 15.6.2010 - C-211/08 -, Kommission/Spanien, NZS 2011, 222; so auch bereits in der Rs. Vanbraekel, EuGH, Urt. v. 12.7.2001 - Rs. C-368/98 -, NZS 2001, 483.

47) Mit dieser sozialpolitisch mehr als abwegig erscheinenden Konsequenz hat offensichtlich die Bundestagsfraktion der Partei „,Die Linke“, die als einzige die Patienten-MobilitätsR L abgelehnt hat, weil von ihr nur Bürger aus wohlhabenden Ländern profitieren könnten, nicht gerechnet. www.linksfraktion.de/reden/gutegesundheitsversorgung-statt-gesundheitsmarkt-in-der-eu.

\title{
Das Recht der biomedizinischen Forschung am Menschen: Nationales Recht im Spiegel internationaler Prinzipien
}

\section{Bernhard Rütsche*}

\section{Internationale Ebene}

\section{Allgemeines}

Seit dem Nürnberger Kodex von 1947 sind auf internationaler Ebene zur Forschung am Menschen im Bereich der Medizin und Biologie zahlreiche Regelwerke in Form von Richtlinien, Deklarationen oder Empfehlungen (Soft Law), aber auch staatsvertraglichen Normen (Hard Law) entstanden. Dabei lassen sich drei Entwicklungsbereiche ausmachen, in denen sich die internationalen - ethischen und rechtlichen - Normen zur Humanforschung herausgebildet haben: ein menschenrechtlicher, ein standesrechtlicher und ein wirtschafts- bzw. industrieorientierter Bereich. Die

Prof. Dr. iur. Bernhard Rütsche,

Ordinarius für öffentliches Recht und Rechtsphilosophie,

Universität Luzern, Rechtswissenschaftliche Fakultät,

Frohburgstraße 3, PF 4466, 6002 Luzern, Schweiz in der Tradition der Menschenrechte stehenden Normen sind primär als Reaktion auf inhumane Experimente zu verstehen und vom obersten Ziel geleitet, die individuellen Versuchspersonen in ihrer Würde und Persönlichkeit zu schützen. Die standesrechtlichen Dokumente sollen Ansehen und Integrität von Ärzten und Wissenschaftlern gewährleisten, was einen hohen ethischen Standard der Forschung notwendig voraussetzt; hinzu kommt das in der Scientific Community hochgehaltene Prinzip einer transparenten und unabhängigen Forschung. Die Arzneimittelund Medizinprodukteindustrie ist demgegenüber in erster Linie an international einheitlichen und günstigen Rahmenbedingungen interessiert. In die Selbstregulierung der Industrie fließen dabei die Anliegen der Zulassungsbehörden ein, welche die Wirksamkeit, Sicherheit und Qualität

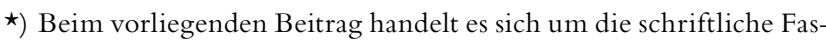
sung eines Vortrags, den der Autor auf der Medizinrechtslehrertagung 2014 in Graz hielt. 
der Produkte im Dienste der öffentlichen Gesundheit zu gewährleisten haben.

\section{Menschenrechtliche Perspektive}

Die Forschung am Menschen ist eines der wichtigsten Themen der jüngeren Menschenrechtsentwicklung auf internationaler Ebene. Die menschenrechtlichen Normtexte im Bereich der Humanforschung sind, wie es für Menschenrechtsdeklarationen typisch ist, meist in Reaktion auf begangene Grausamkeiten und Verfehlungen entstanden. Demzufolge bezwecken diese Normtexte primär den Schutz der in Forschungsprojekte involvierten Personen und die Verhinderung von Forschungsverbrechen. Inhaltlich stehen die Gewährleistung des Selbstbestimmungsrechts, der Schutz von Integrität und Gesundheit der Versuchspersonen sowie der wissenschaftliche und gesellschaftliche Nutzen der Versuche im Zentrum. Da es um den Schutz des Menschen in der Forschung geht - und nicht um die Interessen eines Berufsstandes oder einer Industrie -, sind die menschenrechtlichen Dokumente in ihrem Anwendungsbereich tendenziell weit, d.h. sie beschränken sich nicht auf klinische Versuche mit Arzneimitteln, sondern erfassen auch andere Forschung, die mit Eingriffen in die Integrität des Menschen verbunden ist. Entsprechend ihrem Menschenrechtscharakter handelt es sich sodann weitgehend um Normen mit Grundsatzcharakter.

Der menschenrechtlichen Entwicklungsschiene ist zunächst der Nürnberger Kodex von 1947 mit seinen zehn Prinzipien über ,zulässige medizinische Versuche“ zuzuordnen ${ }^{1}$. Der Nürnberger Kodex entstand als Antwort auf die verbrecherischen Menschenexperimente der Nationalsozialisten und diente als Grundlage für die Verurteilung der angeklagten Ärzte. Der Kodex enthält erstaunlicherweise im Kern schon die allermeisten der heute anerkannten ethischen Grundsätze zur Humanforschung, namentlich die Voraussetzung der informierten Einwilligung in Forschungsversuche (Informed Consent), die Grundsätze der Relevanz und Subsidiarität (Versuche müssen fruchtbare Ergebnisse für das Wohl der Gesellschaft erwarten lassen, welche nicht durch andere Forschungsmittel, z.B. Tierversuche, zu erlangen sind), das Prinzip der Nichtschädigung (Vermeidung unnötiger Schmerzen und Gesundheitsschädigungen sowie vernünftiges Verhältnis zwischen den Gefährdungen für die Versuchspersonen und dem Forschungsziel), die wissenschaftliche Qualifikation der Prüfpersonen sowie das Recht der Versuchspersonen, jederzeit aus dem Versuch auszusteigen.

Der Nürnberger Kodex - das wird manchmal vergessen ist nicht aus dem Nichts entstanden, sondern es gab insbesondere auch in Deutschland Vorläufer, die einzelne Prinzipien, namentlich das Einwilligungserfordernis, bereits vor dem Zweiten Weltkrieg festgeschrieben hatten. Dazu gehört die Anweisung an die Vorsteher der Kliniken, Polikliniken und sonstigen Krankenanstalten des Preussischen Kultusministeriums vom 29.12.1900². Die Anweisung erfolgte vor dem Hintergrund der Versuche zur Serumtherapie gegen Syphilis durch den Breslauer Dermatologen Albert Neisser. Sodann erliess der Deutsche Reichsminister des Innern im Februar 1931 die fortschrittlichen Richtlinien für neuartige Heilbehandlungen und für die Vornahme wissenschaftlicher Versuche am Menschen ${ }^{3}$. Dem Erlass dieser Richtlinien gingen die missglückten Impfversuche in Lübeck (,Lübecker Totentanz“) voraus.

Das bis dahin zwar allgemein anerkannte, aber in der Praxis nicht immer beachtete Erfordernis der freiwilligen Zustimmung zur Teilnahme an medizinischen oder wissenschaftlichen Versuchen wurde 1966 von der UNO im Internationalen Pakt über bürgerliche und politische Rechte im Anschluss an das Folterverbot in Art. 7 S. 2 verankert. Diese Garantie ist maßgeblich geprägt durch die kategori- sche Formulierung von Ziff. 1 des Nürnberger Kodexes zur Einwilligung von Versuchspersonen. Entsprechend ist der Zweck der Garantie darin zu sehen, kriminelle und menschenunwürdige Experimente am Menschen zu verbieten ${ }^{4}$.

In die menschenrechtliche Tradition lässt sich sodann der US-amerikanische Belmont Report vom 30.9.1978 einordnen ${ }^{5}$. Es handelt sich zwar um ein nationales Dokument, das aber internationale Ausstrahlung erlangte und die weitere Entwicklung des Humanforschungsrechts mitprägte. Der Bericht formulierte für die biomedizinische und sozialwissenschaftliche Forschung am Menschen drei grundlegende Prinzipien und deren Ausprägungen: Das Prinzip des Respekts gegenüber der Person (Einwilligung in Forschungsprojekte ohne Zwang und Druck, besonderer Schutz von vulnerablen Personen), das Prinzip der Wohltätigkeit (Schadensvermeidung und Nutzenmaximierung, Risiko/Nutzen-Analyse) sowie das Prinzip der Gerechtigkeit (gleiche Verteilung von Risiken und Nutzen der Forschung sowie faire Auswahl und Behandlung von Studienteilnehmern) ${ }^{6}$. Die Belmont-Kommission wurde mit dem National Research Act von 1974 eingesetzt. Der National Research Act brachte eine strenge Regulierung klinischer Versuche und wurde erlassen, nachdem die Tuskegee-Syphilis-Studie ${ }^{7}$ und andere menschenverachtende Studien an Menschen in den USA bekannt wurden. Der National Research Act sah insbesondere die vorgängige Überprüfung durch Institutional Review Boards auf der Basis von Studienprotokollen vor.

Ein bedeutender, wenn auch in seinen Auswirkungen begrenzter Schritt der Weiterentwicklung der Menschenrechte im Bereich der Biomedizin stellte das Übereinkommen des Europarats über Menschenrechte und Biomedizin vom 4.4.1997 dar. Es handelt sich um einen multilateralen Staatsvertrag, der zwar bisher von 29 Staaten ratifiziert wurde, jedoch bei einigen großen europäischen Staaten

1) Enthalten in der Urteilsbegründung des Nürnberger Ärzteprozesses v. 20.8.1947 vor dem I. amerikanischen Militärgerichtshof; abgedruckt in: Pethes/Griesecke/Krause/Sabisch (Hrsg.), Menschenversuche. Eine Anthologie 1750-2000, 2008, S. 522 f. Zum Rechtsquellencharakter des Nürnberger Kodexes als völkerrechtliches Richterrecht Hohnel, Die rechtliche Einordnung der Deklaration von Helsinki. Eine Untersuchung zur rechtlichen Grundlage humanmedizinischer Forschung, 2005, S. 57; Arnold/Sprumont, Der Nürnberger Kodex: Regeln des Völkerrechts, in: Tröhler/ Reiter=Theil (Hrsg.), Ethik und Medizin 1947-1997: Was leistet die Kodifizierung von Ethik?, 1997, S. $123 \mathrm{f}$.

2) Abgedruckt in: Pethes/Griesecke/Krause/Sabisch (Hrsg.), Menschenversuche. Eine Anthologie 1750-2000, 2008, S. 515.

3) Abgedruckt in: Pethes/Griesecke/Krause/Sabisch (Hrsg.), Menschenversuche. Eine Anthologie 1750-2000, 2008, S. $517 \mathrm{f}$.

4) Vgl. BGE 127 I 6, 16; Sprecher, Medizinische Forschung mit Kindern und Jugendlichen nach schweizerischem, deutschem, europäischem und internationalem Recht, 2007, S. 90; Taupitz, Biomedizinische Forschung zwischen Freiheit und Verantwortung, 2002, S. $117 \mathrm{f}$.

5) Belmont Report: Ethical Principles and Guidelines for the Protection of Human Subjects of Research, Report of the National Commission for the Protection of Human Subjects of Biomedical and Behavioral Research.

6) Ausgehend vom Belmont Report entwickelten Beauchamp und Childress in ihrem Grundlagenwerk „Principles of Biomedical Ethics“ (mittlerweile in der 7. Aufl. 2013) die bis heute anerkannten vier Grundprinzipien der biomedizinischen Ethik: respect for autonomy, nonmaleficence, beneficence, justice. Beauchamp wird eine führende Rolle in der Ausarbeitung des Belmont Report zugeschrieben.

7) Die Studie wurde von 1932 bis 1972 an mit Syphilis infizierten afroamerikanischen Landarbeitern durchgeführt. Der Zweck der Studie bestand darin, den natürlichen Verlauf der SyphilisErkrankung zu beobachten. Die Studie wurde nicht abgebrochen, als wirksame Syphilis-Medikamente erhältlich waren. Die Versuchsteilnehmer hatten keine Gelegenheit zu einer informierten Einwilligung. Sie wurden auch nicht über eine Syphilis-Diagnose unterrichtet. 
nicht auf Akzeptanz gestoßen ist ${ }^{8}$. Das Übereinkommen schreibt in Art. 16 die Mindestvoraussetzungen fest, die für jede Forschung an Personen erfüllt sein müssen, und stellt in Art. 17 besondere Anforderungen an die Forschung mit einwilligungsunfähigen Personen auf. Zur Ergänzung und Konkretisierung dieser Bestimmungen erarbeitete der Europarat das Zusatzprotokoll über biomedizinische Forschung. Dieses auf sämtliche interventionelle Forschung im Gesundheitsbereich anwendbare Zusatzprotokoll wurde jedoch bis 2014 erst von neun Staaten ratifiziert.

Ein mit dem Biomedizin-Übereinkommen des Europarats vergleichbares, wenn auch nicht als Staatsvertrag konzipiertes Dokument stellt die von der Organisation der Vereinten Nationen für Bildung, Wissenschaft und Kultur (UNESCO) am 19.10.2005 verabschiedete Allgemeine Erklärung über Bioethik und Menschenrechte dar. Neben den etablierten ethischen Grundsätzen bezüglich Einwilligung, Risiken und Nutzen, Schutz vulnerabler Personen und unabhängiger Überprüfung durch Ethikkommissionen enthält diese Deklaration insbesondere auch Richtlinien zur Verteilung des Nutzens wissenschaftlicher Forschung (Sharing of Benefits, Art. 15) sowie zur grenzüberschreitenden Forschung (Transnational Practices, Art. 21).

\section{Berufsrecht von Ärzten und Wissenschaftlern}

Vom menschenrechtlichen Ansatz zu unterscheiden ist das internationale Berufs- bzw. Standesrecht von Ärzten und Wissenschaftlern. Der primäre Zweck des Berufsrechts besteht darin, Ansehen, Glaubwürdigkeit und Integrität des Ärztestandes und der Wissenschaft zu wahren. Entsprechend streben die berufs- und standesrechtlichen Normen einen hohen ethischen Standard der Forschung an; zugleich sollen die Anforderungen aber praktikabel bleiben. Das Berufsrecht ist folglich vom Bemühen geleitet, zwischen den Schutzansprüchen betroffener Personen und den Anliegen der Forschungspraxis abzuwägen. Der Forschergemeinschaft (Scientific Community) ist zudem die wissenschaftliche Qualität, Transparenz und Unabhängigkeit der Forschung ein besonderes Anliegen. Die Berufsregeln finden i.S. der Selbstregulierung auf forschende Mediziner Anwendung und sind tendenziell mit dem Anspruch verbunden, als Grundlage oder auch Ersatz für nationale Gesetze zu dienen. Entsprechend ist das Berufsrecht prinzipiell von höherer Normdichte als die menschenrechtlichen Grundsätze, indem relativ konkrete Rechte und Pflichten von Forschenden festgelegt werden.

Die ersten berufsrechtlichen Richtlinien zur Humanforschung auf internationaler Ebene wurden 1954 vom Weltärztebund (World Medical Association, WMA) nach seiner achten Generalversammlung in Rom in Gestalt der Resolution on Human Experimentation: Principles for Those in Research and Experimentation veröffentlicht. Dem 1947 als Zusammenschluss nationaler Ärzteverbände gegründeten Weltärztebund war es wichtig, eine praktikable Forschungsregulierung losgelöst von den Nazi-Verbrechen zu erarbeiten ${ }^{9}$. Die vier Richtlinien der Resolution on $\mathrm{Hu}-$ man Experimentation dienten als Rahmen für die weitere Diskussion und umfassten im Wesentlichen die folgenden Punkte: wissenschaftliche und ethische Qualifikation der Forscher, Zurückhaltung bei der Publikation erster Forschungsresultate, Unterscheidung zwischen Forschung an gesunden und kranken Personen sowie schriftliche Einwilligung in ein Forschungsprojekt.

Auf seiner 18. Generalversammlung in Helsinki im Jahr 1964 beschloss der Weltärztebund die Deklaration „Ethische Grundsätze für die medizinische Forschung am Menschen", die sog. Deklaration von Helsinki. Die Deklaration wurde seither mehrfach revidiert, zuletzt im Jahr 2013 auf der 64. Generalversammlung in Fortaleza, Brasilien. Als Beschluss einer Nichtregierungsorganisation hat die
Helsinki-Deklaration zwar nur empfehlenden Charakter. Durch ihre Inkorporation ins Standesrecht der nationalen Ärztegesellschaften erhält die Deklaration jedoch zivilrechtliche Verbindlichkeit. Als Kodifikation des anerkannten Berufsrechts wirkt die Deklaration zudem auf die Ausgestaltung und Anwendung des nationalen Gesetzesrechts ein. Die breite Akzeptanz der Deklaration zeigt sich auch darin, dass sie in den Präambeln anderer wichtiger Forschungsregulierungen erwähnt wird ${ }^{10}$.

Neben dem Weltärztebund ist der Council for International Organisations of Medical Sciences (CIOMS) ein wichtiger Bestandteil der international vernetzten Scientific Community im Bereich der Biomedizin. Der CIOMS, dessen Mitglieder vor allem nationale Akademien und Kommissionen aus dem Bereich der medizinischen Forschung sind, wurde 1949 als Nichtregierungsorganisation von der Weltgesundheitsorganisation (WHO) und der UNESCO ins Leben gerufen. In den 1970er Jahren wurde der CIOMS beauftragt, Vorschläge darüber auszuarbeiten, wie neue Mitgliedstaaten der WHO - insbesondere Entwicklungsländer - die ethischen Grundsätze der HelsinkiDeklaration umsetzen können. Im Jahr 1982 wurden die Vorschläge in den International Ethical Guidelines for Biomedical Research Involving Human Subjects (CIOMSRichtlinien) kodifiziert und in den Jahren 1993 und 2002 in revidierten Fassungen vorgelegt. Indem die CIOMSRichtlinien darauf angelegt sind, Forschungsmissbräuchen in Entwicklungsländern vorzubeugen, kommt ihnen auch menschenrechtlicher Charakter zu.

Teil der internationalen Wissenschaftsgemeinschaft sind sodann auch die großen Medical Journals. Diese Journals können aufgrund ihrer Marktmacht im Bereich des wissenschaftlichen Verlagswesens normativ auf die Forschungspraxis einwirken, indem sie die Publikation von Forschungsresultaten von gewissen Bedingungen abhängig machen. Ein Beispiel ist die im Jahr 2005 veröffentlichte Ankündigung der wichtigsten Medical Journals, im Interesse der Transparenz der Forschung nur noch klinische Studien zu publizieren, die in Forschungsregistern eingetragen sind ${ }^{11}$.

\section{Selbstregulierung von Industrie und Behörden}

Als dritte Entwicklungsschicht des internationalen $\mathrm{Hu}-$ manforschungsrechts lässt sich die Selbstregulierung von

8) In der Schweiz trat das Übereinkommen am 1.11.2008 in Kraft. Nicht ratifiziert haben das Abkommen namentlich Deutschland, Österreich, Vereinigtes Königreich, Schweden, Italien, Belgien, Irland, Russland und Polen.

9) Lederer, Research Without Borders: The Origins of the Declaration of Helsinki, in: Schmidt/Frewer (Hrsg.), History and Theory of Human Experimentation. The Declaration of Helsinki and Modern Medical Ethics, 2007, S. 203

10) Z.B. Richtlinie 2001/20/EG v. 4.4.2001 zur Angleichung der Rechts- und Verwaltungsvorschriften der Mitgliedstaaten über die Anwendung der guten klinischen Praxis bei der Durchführung von klinischen Prüfungen mit Humanarzneimitteln (EU GCP-Richtlinie), Präambel Ziff. 2; Guideline for Good Clinical Practice der International Conference on Harmonisation (ICH) v. 1.5.1996 (ICH GCP-Richtlinie), Präambel Abs. 1. - Einen gewissen Wirkungsverlust erlitt die Helsinki-Deklaration im Jahr 2008, als die amerikanische Food and Drug Administration (FDA) erklärte, dass sie sich bei der Prüfung von klinischen Versuchen, die im Ausland durchgeführt werden, nicht mehr an den ethischen Grundsätze der Deklaration orientieren werde; vgl. Burgess/Pretorius, FDA abandons the Declaration of Helsinki The effect on the ethics of clinical trial conduct in South Africa and other developing countries, in: South African Journal of Bioethics and Law (SAJBL) 2012, Vol. 5, No. 2, S. $87 \mathrm{fff}$.

11) Abbasi, Compulsory registration of clinical trials, British Medical Journal (BMJ) 2004 S. 637; De Angelis et al., Clinical trial registration: a statement from the International Committee of Medical Journal Editors (ICMJE), The Lancet 2004, 911. 
Industrie und Behörden unterscheiden. Mit der Industrie ist dabei in erster Linie die Pharma- und Medizinprodukteindustrie aus den USA, Europa und Japan gemeint, zu den behördlichen Akteuren gehören Arzneimittelbehörden sowie Behörden der Gesundheits- und Wissenschaftspolitik. Auf den ersten Blick erscheint es überraschend, in Bezug auf staatliche Behörden von „Selbstregulierung“ zu sprechen. Indessen haben sich im Rahmen von Nichtregierungsorganisationen wie der Internationalen Harmonisierungskonferenz (ICH) und der Organisation für wirtschaftliche Zusammenarbeit und Entwicklung (OECD) internationale Kooperationen zwischen Behörden und Industrie bzw. zwischen Behörden allein ergeben, aus denen nichtstaatliches Recht zu klinischen Versuchen hervorgegangen ist. Industrie und Behörden verfolgen dabei das gemeinsame Anliegen, dass die gewonnenen Forschungsdaten glaubwürdig sind, um die Wirksamkeit und Sicherheit der Arzneimittel zu gewährleisten (Validität der Daten). Die Industrie ist zudem an Rechtssicherheit, Verkehrsfähigkeit der internationalen Forschung, fairen Wettbewerbsbedingungen und effizienten Verfahren interessiert, aber auch am guten Ruf und der Akzeptanz der Forschung in der Bevölkerung. Entsprechend konzentrieren sich die Richtlinien von Industrie und Behörden auf die für die Produkteentwicklung erforderlichen klinischen Versuche, wobei die Regelungen einen hohen Detaillierungsgrad aufweisen. Normiert werden insbesondere die ethischen und wissenschaftlichen Qualitätsanforderungen an die Forschung, die Organisation der Forschung (Verantwortlichkeiten von Prüfern, Sponsoren und Auftragsforschungsinstituten), das Qualitätsmanagement (Monitoring, Reporting u.a.) sowie die Überprüfung durch Review Boards bzw. Ethikkommissionen.

Im Zentrum der Selbstregulierung von Industrie und Behörden steht die von der Internationalen Harmonisierungskonferenz erlassene Guideline for Good Clinical Practice vom 1.5.1996 (ICH GCP-Richtlinie). Die Internationale Harmonisierungskonferenz ist eine Nichtregierungsorganisation, die 1989 von Vertretern der nationalen Zulassungsbehörden und der pharmazeutischen Unternehmen aus der EU, den USA und Japan gegründet wurde mit dem Ziel, die Zulassungspraxis für Arzneimittel zu harmonisieren. Die ICH GCP-Richtlinie definiert - nach dem Vorbild der GCP-Richtlinie der US Food and Drug Administration (FDA) von 1977 - einheitliche Vorgaben für die Planung, Durchführung, Dokumentation und Berichterstattung von klinischen Versuchen am Menschen. Sie ist für international operierende Pharmaunternehmen nach wie vor von großer Relevanz ${ }^{12}$, wird von ArzneimittelZulassungsbehörden beachtet und ist von einzelnen Staaten mittels Verweis ins eigene Recht inkorporiert worden ${ }^{13}$. Neben der GCP-Richtlinie existieren zu klinischen und auch nicht-klinischen Prüfungen von Arzneimitteln zahlreiche weitere ICH-Richtlinien mit teilweise stark technischem Inhalt in den vier Bereichen Qualität, Sicherheit, Effizienz und Multidisziplinarität (Quality, Safety, Efficacy and Multidisziplinary Guidelines).

Für Medizinprodukte erfolgt die Zulassung in Europa im Rahmen des sog. New and Global Approach, indem Konformitätsbewertungstellen (Notified Bodies) bzw. die Unternehmen selber die Übereinstimmung der Produkte mit europäisch harmonisierten technischen Normen privater Branchenorganisationen überprüfen. Für klinische Versuche mit Medizinprodukten hat die International Organization for Standardization (ISO) im Jahr 2011 Good Clinical Practice Normen erlassen ${ }^{14}$.

Zur Selbstregulierung im Forschungsrecht sind neben den erwähnten GCP-Normen auch die Verhaltens-Kodizes der Pharmaunternehmen und ihrer Verbände zu zählen. Diese Kodizes enthalten neben wettbewerbsrechtlichen Themen im Zusammenhang mit der Werbung und dem Arzneimittelvertrieb auch ethische Richtlinien, welche unter anderem die klinische Forschung betreffen, namentlich die Finanzierung der Forschung, die Zusammenarbeit mit Prüfpersonen und Gesundheitsinstitutionen sowie die Offenlegung von geldwerten Leistungen. Auf internationaler Ebene haben die International Federation of Pharmaceutical Manufactueres \& Associations (IFPMA), der Verband der weltweit agierenden pharmazeutischen Industrie, sowie die European Federation of Pharmaceutical Industries and Associations (EFPIA), der Dachverband der nationalen Pharmaverbände in Europa sowie einzelner pharmazeutischer Unternehmen, Verhaltens-Kodizes für die Branche verfasst ${ }^{15}$. Diese internationalen Branchenregulierungen werden durch die nationalen Pharmaverbände implementiert und durchgesetzt. Was den Code of Pratice der IFPMA betrifft, erfolgt die Durchsetzung zudem subsidiär durch das IFPMA-Sekretariat, das ein regelwidriges Verhalten mittels Publikation des Falls im Internet unter Bekanntgabe der Identität des fehlbaren Unternehmens und der relevanten Fakten sanktionieren kann ${ }^{16}$.

Als weiteres Beispiel internationaler Selbstregulierung im Bereich der Humanforschung lässt sich schließlich die Recommendation on the Governance of Clinical Trials der OECD vom 10.12.2012 aufführen. Dieses nicht-bindende Dokument wurde vom Global Science Forum ausgearbeitet, einer OECD-Plattform für die Zusammenarbeit hoher Funktionäre der Mitgliedstaaten auf dem Gebiet der Wissenschafts- und Technologiepolitik. Die OECDEmpfehlung soll zu einer Harmonisierung der nationalen Regulierungen klinischer Versuche beitragen, indem sie den Mitgliedstaaten einen risikobasierten Ansatz empfiehlt, welcher insbesondere bei der vorgängigen Überprüfung klinischer Versuche durch staatliche Arzneimittelbehörden zum Tragen kommen soll.

\section{Universelle Prinzipien des Humanforschungsrechts}

\section{Allgemeines}

Die internationalen Regelwerke zur Humanforschung sind im Wesentlichen auf zwei fundamentale Antriebskräfte zurückzuführen: einerseits auf das ethische Streben nach einer gerechten Ordnung oder zumindest einer Verhinderung von Grausamkeiten, wobei dieses Interesse intrinsischer Art sein kann, unter Umständen aber auch von der Sorge der Forschenden um gute Reputation herrührt; anderseits auf das ökonomische Anliegen gleicher und günstiger Rahmenbedingungen für die Entfaltung eines grenzüberschreitenden Wettbewerbs, sei es zur Steigerung der allgemeinen Wohlfahrt, sei es zur Optimierung unternehmerischer Gewinnmöglichkeiten. Vor dem Hintergrund, dass die Ethik zu den Interessen der Wirtschaft zumindest prima facie in einem Spannungsfeld steht, mag es überraschen, dass zwischen den menschenrechtlichen, berufsrechtlichen und wirtschaftsorientierten Normtexten weitgehende in-

12) Herschel, Praxisbuch Klinische Forschung, 2. Aufl. 2013, S. 39.

13) Z.B. in der Schweiz: Verordnung v. 20.9.2013 über klinische Versuche in der Humanforschung (Verordnung über klinische Versuche; KlinV; SR 810.305), Anhang 1 Ziff. 2.

14) Norm EN ISO 14155:2011.

15) IFPMA Code of Practice, aktuelle Version v. 2012; EFPIA Code of Practice on the Promotion of Prescription-only Medicines to, and Interactions with Healthcare Professionals (EFPIA HCP Code), aktuelle Version v. 2014; EFPIA Code on Disclosure of Transfers of Value from Pharmaceutical Companies to Healthcare Professionals and Healthcare Organisations (EFPIA Disclosure Code) v. 2014.

16) IFPMA Code of Practice 2012, Appendix 1, insbesondere Ziff. 2.8 . 
haltliche Konvergenzen festgestellt werden können. So hat sich im internationalen Humanforschungsrecht ein Grundbestand von Prinzipien mit jeweiligen Konkretisierungen herausgebildet, welche in mehreren, teilweise sogar allen Normtexten verankert sind. Diese Prinzipien weisen über die Zeit hinweg eine hohe Beständigkeit auf, haben in verschiedenen regulatorischen Kontexten Akzeptanz gefunden und sind weltweit oder zumindest in den Industrienationen mit eigener biomedizinischer Forschung verbreitet. Insofern kann den Prinzipien universalistischer Charakter zugesprochen werden. Im Folgenden werden diese Prinzipien und ihre Konkretisierungen kurz dargestellt, wobei als Quellen die wichtigsten Regelwerke heranzuziehen sind: der Nürnberger Kodex, das Biomedizin-Übereinkommen, die Allgemeine Erklärung über Bioethik und Menschenrechte, die Helsinki-Deklaration, die CIOMS-Richtlinien und die ICH GCP-Richtlinie.

\section{Einzelne Prinzipien}

a) Informed Consent

Das Prinzip, dass kein Forschungsprojekt ohne explizite vorgängige Einwilligung der teilnehmenden Personen durchgeführt werden darf, ist in sämtlichen internationalen Richtlinien und Staatsverträgen zur biomedizinischen Forschung verankert. Dabei wird regelmäßig eine schriftliche Dokumentation der Einwilligung verlang $\mathrm{t}^{17}$. Der Einwilligung hat eine angemessene Aufklärung insbesondere über Zweck, Methode und Mittel des Forschungsprojekts, die Risiken und potenziellen Belastungen sowie die Rechte der Versuchspersonen voranzugehen ${ }^{18}$. Die Einwilligung muss auf Freiwilligkeit beruhen, d.h. unbeeinflusst von Zwang, Druck, Täuschung oder Überredung erfolgen ${ }^{19}$. Zur Sicherstellung der Freiwilligkeit wird teilweise auch ein Verbot übermäßiger finanzieller Entgelte, welche Personen zur Teilnahme an Forschungsprojekten verleiten können, festgelegt ${ }^{20}$. Universeller Natur ist sodann das Recht von Versuchspersonen, die Einwilligung in die Teilnahme an einem Forschungsprojekt jederzeit $\mathrm{zu}$ widerrufen und aus dem Projekt auszusteigen ${ }^{21}$.

\section{b) Verhältnismäßigkeit der Risiken und Belastungen}

Zum Schutz der physischen und psychischen Integrität der Versuchspersonen verlangen die internationalen Richtlinien und Verträge in verschiedener Hinsicht, dass die mit der Teilnahme an Forschungsprojekten verbundenen Risiken und Belastungen verhältnismäßig sind. Dazu gehört zunächst die Pflicht von Prüfpersonen und Sponsoren, die Risiken und Belastungen vor dem Versuch zu analysieren und während der Durchführung des Versuchs zu beobachten und $\mathrm{zu}$ dokumentieren (Risiko-Beurteilung) ${ }^{22}$. Dabei sind die Risiken und Belastungen mittels geeigneter Massnahmen so gering wie möglich zu halten, und für den Fall unerwünschter Gesundheitsschädigungen müssen die betroffenen Versuchspersonen angemessen versorgt und behandelt werden (Risiko-Vermeidung) ${ }^{23}$. Fest etabliert ist alsdann die Grundregel, dass der zu erwartende Nutzen eines Forschungsprojekts für die Wissenschaft und die Gesellschaft ein hinreichendes Gewicht haben muss, um die mit dem Projekt voraussichtlich verbundenen Risiken und Belastungen zu rechtfertigen (Risiko/Nutzen-Abwägung) ${ }^{24}$. Für Placebo-kontrollierte Studien wird die Risiko/Nutzen-Abwägung im Hinblick auf die Gefahr von Gesundheitsschädigungen infolge von Nichtbehandlung teilweise spezifiziert ${ }^{25}$. In einzelnen Dokumenten findet sich zudem die allgemeine Formulierung, dass die Rechte und Interessen der Individuen Vorrang gegenüber den Kollektivinteressen von Wissenschaft und Gesellschaft haben ${ }^{26}$; die praktische Bedeutung dieses Prinzips zeigt sich wohl vor allem darin, dass Forschungsexperimente abzubrechen sind, wenn sich herausstellt, dass die Risiken und Belastun- gen größer als erwartet und für die Forschungsteilnehmenden nicht mehr zumutbar sind ${ }^{27}$.

c) Besonderer Schutz vulnerabler Personen

In die biomedizinische Forschung werden auch Personen einbezogen, deren Autonomie beschränkt ist und die deshalb besonders verletzlich sind. Dazu gehören Kinder, urteilsunfähige Erwachsene, Notfallpatienten wie auch Gefängnis- und Anstaltsinsassen. Soweit diese Personen einwilligungsunfähig sind, verlangen die internationalen Dokumente die informierte Einwilligung durch einen gesetzlichen Stellvertreter ${ }^{28}$. In neueren Normtexten wird, in Anlehnung an die UN-Konvention über die Rechte des Kindes von 1989 (Art. 12 Abs. 1), gefordert, dass Einwilligungsunfähige an der Entscheidfindung partizipieren, wobei der Meinung minderjähriger Personen mit zunehmendem Alter und zunehmender Reife immer mehr entscheidendes Gewicht zukommen soll ${ }^{29}$; häufig wird Einwilligungsunfähigen auch das Recht eingeräumt, die Teilnahme an Forschungsprojekten abzulehnen ${ }^{30}$. Zum Schutz der Integrität vulnerabler Personen statuieren sodann verschiedene Regelwerke ein Subsidiaritätsprinzip: Danach dürfen vulnerable Personen nur dann in ein Forschungsprojekt einbezogen werden, wenn Forschung von vergleichbarer Wirksamkeit an einwilligungsfähigen Personen nicht möglich ist ${ }^{31}$. Für

17) Helsinki-Deklaration Ziff. 26; CIOMS-Richtlinien Ziff. 6; ICH GCP-Richtlinie Ziff. 4.8.

18) Nürnberger Kodex Ziff. 1; Biomedizin-Übereinkommen Art. 16; Allgemeine Erklärung über Bioethik und Menschenrechte Art. 6; Helsinki-Deklaration Ziff. 26; CIOMS-Richtlinien Ziff. 5; ICH GCP-Richtlinie Ziff. 4.8.

19) Nürnberger Kodex Ziff. 1; Biomedizin-Übereinkommen Art. 16 (i. V. mit Art. 5); Allgemeine Erklärung über Bioethik und Menschenrechte Art. 6 (vgl. auch Art. 15 Abs. 2); Helsinki-Deklaration Ziff. 25, 27; CIOMS-Richtlinien Ziff. 4 und 6; ICH GCPRichtlinie Ziff. 2.9, 4.8.

20) CIOMS-Richtlinien Ziff. 7; ICH GCP-Richtlinie Ziff. 3.1.8.

21) Nürnberger Kodex Ziff. 9; Biomedizin-Übereinkommen Art. 16; Allgemeine Erklärung über Bioethik und Menschenrechte Art. 6; Helsinki-Deklaration Ziff. 28, 31; CIOMS-Richtlinien Ziff. 5; ICH GCP-Richtlinie Ziff. 4.8.

22) Helsinki-Deklaration Ziff. 17; ICH GCP-Richtlinie Ziff. 4.10, 5.18, 6.2.3. Vgl. auch Allgemeine Erklärung über Bioethik und Menschenrechte Art. 20.

23) Nürnberger Kodex Ziff. 4 und 7; Allgemeine Erklärung über Bioethik und Menschenrechte Art. 4; Helsinki-Deklaration Ziff. 15, 17; CIOMS-Richtlinien Ziff. 8, 19, 21; ICH GCPRichtlinie Ziff. 4.3.

24) Nürnberger Kodex Ziff. 6; Biomedizin-Übereinkommen Art. 16; Helsinki-Deklaration Ziff. 16; CIOMS-Richtlinie Ziff. 8; ICH GCP-Richtlinie Ziff. 2.2.

25) Helsinki-Deklaration Ziff. 33; CIOMS-Richtlinie Ziff. 11.

26) Biomedizin-Übereinkommen Art. 2; Allgemeine Erklärung über Bioethik und Menschenrechte Art. 3 Abs. 2; Helsinki-Deklaration Ziff. 8; ICH GCP-Richtlinie Ziff. 2.3.

27) Vgl. Nürnberger Kodex Ziff. 10; Helsinki-Deklaration Ziff. 18.

28) Biomedizin-Übereinkommen Art. 6 Abs. 2 und 4; Helsinki-Deklaration Ziff. 28, 30; CIOMS-Richtlinien Ziff. 4, 14, 15; ICH GCP-Richtlinie Ziff. 4.8 .

29) Biomedizin-Übereinkommen Art. 6 Abs. 2 und 3; Allgemeine Erklärung über Bioethik und Menschenrechte Art. 7; HelsinkiDeklaration Ziff. 29.

30) Biomedizin-Übereinkommen Art. 17 Abs. 1; Allgemeine Erklärung über Bioethik und Menschenrechte Art. 7; HelsinkiDeklaration Ziff. 29; CIOMS-Richtlinie Ziff. 14, 15.

31) Biomedizin-Übereinkommen Art. 17 Abs. 1; Allgemeine Erklärung über Bioethik und Menschenrechte Art. 7 (nur in Bezug auf Forschung ohne erwarteten direkten Nutzen); Helsinki-Deklaration Ziff. 28 (nur in Bezug auf Forschung ohne erwarteten direkten Nutzen); Helsinki-Deklaration Ziff. 20; CIOMS-Richtlinien Ziff. 13 (vulnerable Personen), 14 (Kinder), 15 (Einwilligungsunfähige), 17 (schwangere Frauen); ICH GCP-Richtlinie Ziff. 4.8.14 (nur in Bezug auf Forschung ohne erwarteten direkten Nutzen). 
rein fremdnützige Forschungsprojekte, d.h. Projekte, deren erwartete Ergebnisse für die Gesundheit der Versuchspersonen nicht von direktem Nutzen sind, werden erhöhte Schutzanforderungen aufgestellt: Einwilligungsunfähige Personen dürfen nur dann in solche Projekte einbezogen werden, wenn die Forschung minimale Risiken und Belastungen mit sich bringt und einen Gruppennutzen erwarten lässt, d.h. Personen nützen kann, welche derselben Kategorie (insbesondere hinsichtlich Alter, Krankheit oder Störung) angehören ${ }^{32}$.

\section{d) Wissenschaftlicher Nutzen der Forschung}

Eingriffe in die Integrität von Personen zu Forschungszwecken können nur gerechtfertigt werden, wenn die Forschung von wissenschaftlichem Wert ist und damit einen allgemeinen Nutzen generiert. Wissenschaftlich unbrauchbare Forschung ist unethische Forschung. Diese Einsicht ist in internationalen Normtexten breit verankert ${ }^{33}$. Im Einzelnen wird insbesondere verlangt, dass die fachliche Qualifikation der Forschenden sichergestellt ist ${ }^{34}$. Damit Forschungsprojekte für die Wissenschaft einen Nutzen bringen und praktisch verwertet werden können, bedarf es zudem der Transparenz. Die Helsinki-Deklaration fordert entsprechend, dass Forschungsprojekte am Menschen im Bereich der Medizin vor ihrem Beginn in einer öffentlichen Datenbank registriert ${ }^{35}$ und deren Resultate - positive wie negative - nach der Durchführung und Auswertung publiziert werden ${ }^{36}$. Transparenzbestimmungen finden sich auch in den internationalen Pharma-Kodizes ${ }^{37}$, und verschiedene Medical Journals setzen für die Publikation von Forschungsresultaten wie erwähnt voraus, dass die Forschungsprojekte in einem öffentlichen Register eingetragen worden sind ${ }^{38}$. Dennoch ist das internationale Forschungsrecht in Transparenzfragen in Entwicklung begriffen, und es lassen sich noch kaum fest etablierte und breit anerkannte Grundsätze feststellen.

\section{e) Unabhängige Überprüfung}

Ein fester Bestandteil des internationalen Humanforschungsrechts ist das Prinzip, dass Forschungsprojekte vor ihrer Durchführung von einer unabhängigen Stelle überprüft werden ${ }^{39}$. Diese Stelle wird als ,Research Ethics Committee“, „Ethical Review Committee“, „Review Board" oder anderswie bezeichnet. Auf Deutsch ist der Ausdruck „Ethikkommission“ geläufig. Ob Ethikkommissionen mit Hoheitsgewalt ausgestattet sind und verbindliche Entscheide treffen können oder als nicht-staatliche Einrichtungen Stellungnahmen abgeben, wird von den internationalen Normen nicht vorgegeben; entscheidend ist die Unabhängigkeit der Kommissionen gegenüber den Forschenden und den Sponsoren der Forschung. Einzelne Richtlinien sehen zudem vor, dass Ethikkommissionen Forschungsprojekte soweit notwendig auch während ihrer Durchführung beaufsichtigen ${ }^{40}$.

\section{f) Weitere Prinzipien}

Die erwähnten Prinzipien sind für den Schutz der Autonomie und Integrität von Versuchspersonen in der biomedizinischen Forschung von überragender Bedeutung. Darüber hinaus lassen sich weitere Prinzipien ausmachen, die in mehreren internationalen Regelwerken verankert sind. Breit anerkannt ist das Prinzip der Vertraulichkeit von Personendaten, die im Rahmen von Forschungsprojekten erhoben werden ${ }^{41}$. Sodann ist in einzelnen Dokumenten die Subsidiarität der Humanforschung explizit festgeschrieben. Demzufolge ist Forschung am Menschen nur zulässig, wenn keine anderen Forschungsmittel oder Methoden von vergleichbarer Wirksamkeit existieren ${ }^{42}$. Weiter findet sich die Pflicht zur finanziellen Kompensation von Personen, die infolge der Teilnahme an einem Forschungsprojekt einen Schaden erlitten haben ${ }^{43}$, wobei die ICH GCP-
Richtlinien diesbezüglich auf die anwendbaren nationalen Regulierungen verweisen ${ }^{44}$. Schließlich verlangen die Helsinki-Deklaration und die CIOMS-Richtlinien, dass unterrepräsentierte Gruppen angemessen in die Forschung einbezogen werden ${ }^{45}$.

\section{Verwirklichung im schweizerischen Recht}

\section{Allgemeines}

Die internationalen Vorgaben zur biomedizinischen Forschung am Menschen stellen zu einem großen Teil unverbindliche Empfehlungen (Soft Law) dar, die der Umsetzung ins nationale bzw. europäische Recht bedürfen, damit sie rechtlich durchgesetzt werden können. Teilweise werden die Richtlinien ins nationale Standesrecht der Ärzte bzw. in die nationalen Selbstregulierungen der Pharmabranche übernommen, sodass sie für die Mitglieder dieser Organisationen zivilrechtliche Verbindlichkeit erlangen. Eine allgemeine - öffentlich-rechtliche - Verbindlichkeit erhalten die Richtlinien erst dadurch, dass das nationale Recht auf sie verweist oder sie inhaltlich nachvollzieht. Angesichts der breiten Akzeptanz der dargestellten - als universell bezeichneten - Prinzipien, ihrer Bedeutung mit Blick auf einheitliche Rahmenbedingungen für die grenzüberschreitende klinische Forschung und ihrer Etablierung in der Forschungspraxis besteht für den Gesetzgeber ein gewisser moralischer und auch ökonomischer Druck, diese Prinzipien ins nationale Recht zu überführen. AnschlieBend wird aufgezeigt, wie dies im Fall der Schweiz geschehen ist. Die Schweiz bietet sich deshalb als Beispiel an, weil dort das Recht der Forschung am Menschen auf Anfang 2014 eine umfassende Neuordnung erfahren hat, welche mit einer kritischen Rezeption der internationalen Vorgaben verbunden war $^{46}$.

\section{Verfassungsartikel über die Forschung am Menschen}

Die Grundlage für die Kodifikation des Humanforschungsrechts in der Schweiz wurde mit dem am 7.3.2010

32) Biomedizin-Übereinkommen Art. 17 Abs. 2; Allgemeine Erklärung über Bioethik und Menschenrechte Art. 7; Helsinki-Deklaration Ziff. 28. Vgl. auch ICH GCP-Richtlinie Ziff. 4.8.14.

33) Nürnberger Kodex Ziff. 2; Biomedizin-Übereinkommen Art. 16; Helsinki-Deklaration Ziff. 6; CIOMS-Richtlinien 1; ICH GCP-Richtlinie Ziff. 2.5.

34) Nürnberger Kodex Ziff. 8; Helsinki-Deklaration Ziff. 12; ICH GCP-Richtlinie Ziff. 2.8, 4.1.

35) Helsinki-Deklaration Ziff. 35

36) Helsinki-Deklaration Ziff. 36

37) Vgl. Junod, Transparence dans la recherche médicale, jusletter 2014, Rdnr. 2 m. w. Hinw.

38) S. oben, sub I.3. a.E.

39) Biomedizin-Übereinkommen Art. 16; Allgemeine Erklärung über Bioethik und Menschenrechte Art. 19; Helsinki-Deklaration Ziff. 23; CIOMS-Richtlinien Ziff. 2; ICH GCP-Richtlinie Ziff. 2.6, 3.1.

40) Helsinki-Deklaration Ziff. 23; CIOMS-Richtlinien Ziff. 2; ICH GCP-Richtlinie Ziff. 3.1.4.

41) Allgemeine Erklärung über Bioethik und Menschenrechte Art. 9; Helsinki-Deklaration Ziff. 9, 24; CIOMS-Richtlinien Ziff. 18; ICH GCP-Richtlinie Ziff. 2.11, 4.8.10. Vgl. auch Biomedizin-Übereinkommen Art. 10 Abs 1.

42) Nürnberger Kodex Ziff. 2; Biomedizin-Übereinkommen Art. 16.

43) Helsinki-Deklaration Ziff. 15; CIOMS-Richtlinien Ziff. 19

44) ICH GCP-Richtlinie Ziff. 5.8.

45) Helsinki-Deklaration Ziff. 13; CIOMS-Richtlinien Ziff. 12, 16 (betreffend Frauen).

46) Dazu Rütsche, Die Neuordnung des schweizerischen Humanforschungsrechts: Normgenese als kritische Rezeption internationaler Vorgaben, in: ZSR 2010 I $391 \mathrm{ff}$. 
von Volk und Ständen angenommenen Verfassungsartikel über die Forschung am Menschen, Art. 118b BV ${ }^{47}$, gelegt. Mit dem Verfassungsartikel erhielt der Bund den Auftrag und die Kompetenz, Vorschriften über die Forschung am Menschen zu erlassen, ,,soweit der Schutz seiner Würde und seiner Persönlichkeit es erfordert". Es handelt sich um eine umfassende Kompetenz, die unabhängig von der wissenschaftlichen Disziplin sämtliche Forschung am Menschen einschließt. Allerdings darf der Gesetzgeber die Forschung nur soweit regulieren, als von ihr eine Gefährdung der Würde und Persönlichkeit des Menschen ausgeht; diese gefährdungsbezogene Konzeption steht im Dienst der Forschungsfreiheit und soll eine Überregulierung der Forschung verhindern ${ }^{48}$.

Um das Humanforschungsrecht auf eine solide Legitimationsgrundlage zu stellen, die in der Bevölkerung Akzeptanz schafft und Vertrauen bildet, beschränkte sich der Verfassungsgeber nicht auf eine reine Kompetenznorm, sondern gab dem Gesetzgeber zugleich die wichtigsten forschungsrechtlichen Grundsätze vor. Diese Grundsätze sind in Art. 118b Abs. 2 BV statuiert und beziehen sich auf „Forschung in Biologie und Medizin mit Personen“, also auf interventionelle Forschung im Bereich der Biomedizin. Inhaltlich widerspiegeln die Grundsätze nahezu exakt die dargelegten universellen Prinzipien des $\mathrm{Hu}-$ manforschungsrechts: In Buchstabe a wird das Erfordernis der Einwilligung nach hinreichender Aufklärung festgeschrieben, wobei der Gesetzgeber Ausnahmen festlegen kann; eine Ablehnung der Teilnahme an der Forschung ist indessen in jedem Fall zu beachten. Buchstabe b hält fest, dass die Risiken und Belastungen für die teilnehmenden Personen nicht in einem Missverhältnis zum Nutzen des Forschungsvorhabens stehen dürfen. Buchstabe c verankert das Subsidiaritätsprinzip für Forschung mit urteilsunfähigen Personen; diese darf nur durchgeführt werden, wenn gleichwertige Erkenntnisse nicht mit urteilsfähigen Personen gewonnen werden können. Zudem wird festgehalten, dass die Risiken und Belastungen nur minimal sein dürfen, wenn das Forschungsvorhaben keinen unmittelbaren Nutzen für die urteilsunfähigen Personen erwarten lässt. Schließlich verlangt Buchstabe d, dass jẹdes Forschungsvorhaben vorgängig einer unabhängigen Überprüfung bedarf.

\section{Gesetz über die Forschung am Menschen}

Gestützt auf Art. 118b Abs. 1 BV erließ der Bundesgesetzgeber am 30.9.2011 das Gesetz über die Forschung am Menschen (Humanforschungsgesetz, $\mathrm{HFG}^{49}$ ). Das Gesetz trat auf den 1.1.2014 zusammen mit drei Regierungsverordnungen $^{50}$ in Kraft. Es gilt nicht nur für biomedizinische Forschung am Menschen, sondern für sämtliche „Forschung zu Krankheiten des Menschen sowie zu Aufbau und Funktion des menschlichen Körpers" ${ }^{\text {"51 }}$; darin eingeschlossen ist die ,Grundlagenforschung, insbesondere zur Anatomie, Physiologie und Genetik des menschlichen Körpers, sowie nicht auf Krankheiten bezogene Forschung $\mathrm{zu}$ Eingriffen und Einwirkungen auf den menschlichen Körper" ${ }^{\circ 52}$.

Das Gesetz über die Forschung am Menschen verwirklicht und konkretisiert in umfassender Weise die universellen Prinzipien des Humanforschungsrechts sowie die Grundsätze des Verfassungsartikels über die Forschung am Menschen. Was den Grundsatz des Informed Consent betrifft, ist die in Art. 18 HFG vorgesehene Ausnahme von der vollständigen Aufklärung bemerkenswert. Danach darf die betroffene Person über einzelne Punkte eines Forschungsprojekts vor dessen Beginn nur partiell aufgeklärt werden, soweit dies aus methodischen Gründen zwingend ist und mit dem Forschungsprojekt nur minimale Risiken und Belastungen verbunden sind. Die teilnehmende Person muss indessen nachträglich so bald als möglich hinreichend aufgeklärt werden, wobei sie die Verwendung ihres biologischen Materials oder ihrer Personendaten für das Forschungsprojekt untersagen kann. Erwähnenswert ist sodann die Regelung finanzieller Anreize. Gemäß Art. 14 Abs. $1 \mathrm{HFG}$ darf niemand für die Teilnahme an einem Forschungsprojekt mit erwartetem direktem Nutzen ein Entgelt oder einen andern geldwerten Vorteil erhalten; die Teilnahme an einem Forschungsprojekt ohne erwarteten direkten Nutzen kann indessen angemessen entgolten werden.

Die Anforderungen an die Verhältnismäßigkeit der Risiken und Belastungen werden in verschiedener Hinsicht umgesetzt. Unter den Grundsätzen schreibt das Gesetz in allgemeiner Weise fest, dass Interesse, Gesundheit und Wohlergehen des einzelnen Menschen Vorrang haben gegenüber den Interessen der Wissenschaft und der Gesellschaft ${ }^{53}$. Art. 12 Abs. $1 \mathrm{HFG}$ verlangt, dass bei jedem Forschungsprojekt die Risiken und Belastungen für die teilnehmenden Personen so gering wie möglich gehalten werden. Entsprechend haben Forschende vor Projektbeginn wie auch während der Durchführung des Projekts alle erforderlichen Schutzmaßnahmen zu treffen, falls Umstände auftreten, welche die Sicherheit oder die Gesundheit der Versuchspersonen beeinträchtigen können oder die zu einem Missverhältnis zwischen den Risiken und Belastungen und dem Nutzen führen ${ }^{54}$. Die bereits auf Verfassungsstufe verankerte Risiko/Nutzen-Abwägung wird in Art. 12 Abs. 2 HFG wiederholt und in Art. 13 HFG in Bezug auf Placebo-kontrollierte Studien konkretisiert.

Für die Forschung mit vulnerablen Personen - in der Terminologie des Humanforschungsgesetzes ,besonders verletzbaren Personen“ - gelten zusätzliche Anforderungen, die sich ebenfalls an den internationalen Richtlinien orientieren. So gewährleistet das Gesetz Partizipations- und Ablehnungsrechte von Kindern ${ }^{55}$, Jugendlichen ${ }^{56}$ und urteilsunfähigen Erwachsenen ${ }^{57}$. Das Subsidiaritätsprinzip wird in Art. 11 Abs. 2 HFG in Bezug auf alle besonders verletzbaren Personen statuiert; zu diesen gehören unabhängig von ihrer Urteilsfähigkeit sämtliche Kinder und Jugendliche (d.h. Minderjährige), urteilsunfähige Erwachsene, Notfallpatienten, Personen im Freiheitsentzug sowie schwangere Frauen und Embryonen bzw. Föten in vivo. Die Voraussetzungen der minimalen Risiken und Belastungen sowie des Gruppennutzens gelten für alle Forschung ohne erwarteten direkten Nutzen mit urteilsunfähigen Personen ${ }^{58}$.

47) Bundesverfassung der Schweizerischen Eidgenossenschaft v. 18. 4. 1999 (SR 101).

48) Schweizerischer Bundesrat, Botschaft v. 12.9.2007 zum Verfassungsartikel über die Forschung am Menschen, BBl 2007 6721, 6730 .

49) SR 810.30

50) Verordnung v. 30.9.2013 über die Humanforschung mit Ausnahme der klinischen Versuche (Humanforschungsverordnung, HFV; SR 810.301); Verordnung v. 30.9.2013 über klinische Versuche in der Humanforschung (Verordnung über klinische Versuche; KlinV; SR 810.305); Organisationsverordnung v. 30.9.2013 zum Humanforschungsgesetz (Organisationsverordnung HFG, OV-HFG; SR 810.308).

51) Art. 2 Abs. 1 HFG.

52) Art. 3 Bst. c HFG.

53) Art. 4 HFG.

54) Art. 15 HFG.

55) Art. 21, Art. 22 Abs. 3 Buchst. b HFG.

56) Art. 21, Art. 23 Abs. 2 Buchst. b HFG.

57) Art. 21 Abs. 1, Art. 24 Abs. 1 Buchst. c HFG. Vgl. auch Art. 30 Abs. 1 Buchst. b HFG in Bezug auf Notfallpatienten.

58) Art. 22 Abs. 2, Art. 23 Abs. 3, Art. 24 Abs. 2, Art. 30 Abs. 2 HFG. 
Der Grundsatz, dass die Humanforschung einen wissenschaftlichen Nutzen mit sich bringen muss, kommt zunächst in Art. 5 HFG zum Ausdruck, wonach Forschung am Menschen nur durchgeführt werden darf, wenn eine wissenschaftlich relevante Fragestellung gegeben ist. Sodann legt Art. 10 HFG die wissenschaftlichen Anforderungen an Forschungsprojekte fest. Danach darf Forschung am Menschen nur durchgeführt werden, wenn die anerkannten Regelungen über die wissenschaftliche Integrität eingehalten werden (insbesondere bezüglich des Umgangs mit Interessenkonflikten), die Anforderungen an die wissenschaftliche Qualität erfüllt sind, die anerkannten internationalen Regeln der Guten Praxis über die Forschung am Menschen eingehalten werden und die verantwortlichen Personen fachlich hinreichend qualifiziert sind. Was die Transparenz der Forschung angeht, führt Art. 56 Abs. 1 HFG eine Registrierungspflicht für bewilligte klinische Versuche ein. Demgegenüber hat der Gesetzgeber darauf verzichtet, eine Pflicht zur Publikation von Forschungsresultaten festzuschreiben.

Der bereits in der Verfassung vorgesehene Grundsatz der unabhängigen Überprüfung wird in Artt. $45 \mathrm{ff}$. HFG umgesetzt. Gemäß Art. 45 Abs. 1 HFG bedarf es für die Durchführung eines Forschungsprojekts einer Bewilligung der zuständigen Ethikkommission, welche überprüft, ob die ethischen, rechtlichen und wissenschaftlichen Anforderungen des Gesetzes erfüllt sind. Die Ethikkommissionen sind kantonale Einrichtungen, welche vom Gesetzgeber mit staatlicher Hoheitsgewalt ausgestattet werden. Deren Entscheide sind verbindliche Verfügungen, die auf dem verwaltungsrechtlichen Rechtsweg bis an das Bundesgericht angefochten werden können ${ }^{59}$. Zudem haben die Ethikkommissionen gewisse Aufsichtskompetenzen über laufende Forschungsprojekte. Sie können von Bewilligungsinhabern Auskünfte und Unterlagen verlangen und als ultima ratio mittels Widerrufs der Bewilligung ein Forschungsprojekt abbrechen, wenn die Sicherheit oder Gesundheit der betroffenen Personen gefährdet ist ${ }^{60}$.

Erwähnt sei weiter, dass das schweizerische Humanforschungsrecht den von der OECD empfohlenen ${ }^{61}$ risikobasierten Ansatz umsetzt. So verlangt Art. 65 Abs. 2 HFG, dass der Bundesrat beim Erlass des ausführenden Verordnungsrechts das unterschiedliche Ausmaß der Gefährdung von Würde und Persönlichkeit, das mit den einzelnen Forschungsbereichen und -vorgehen verbunden ist, beachtet. Gestützt darauf hat der Bundesrat namentlich klinische Versuche mit Arzneimitteln und Medizinprodukten in drei Gefährdungskategorien eingeteilt ${ }^{62}$ und klinische Versuche der tiefsten Gefährdungskategorie (Kategorie A) von der Bewilligungspflicht beim schweizerischen Heilmittelinstitut (Swissmedic) ausgenommen ${ }^{63}$.

Schließlich haben auch weitere international anerkannte Prinzipien ihren Niederschlag im Humanforschungsgesetz gefunden. Dazu gehört das allgemeine Subsidiaritätsprinzip, wonach ein Forschungsprojekt mit Personen nur durchgeführt werden darf, wenn gleichwertige Erkenntnisse anders nicht gewonnen werden können ${ }^{64}$. Der Anspruch von Versuchspersonen auf finanzielle Kompensation für Schäden, die sie im Zusammenhang mit einem Forschungsprojekt erleiden, wird in Art. 19 Abs. 1 HFG in Form einer verschuldensunabhängigen Gefährdungshaftung verankert; haftbar ist der Sponsor, d.h. die Person, welche die Durchführung des Projekts veranlasst hat. Ferner statuiert Art. 6 HFG ein Diskriminierungsverbot im Rahmen der Forschung. Nach Absatz 2 dieser Bestimmung darf insbesondere bei der Auswahl der Personen für die Forschung keine Personengruppe ohne triftige Grün- de übermäßig in die Forschung einbezogen oder von der Forschung ausgeschlossen werden. Eigentliche Ansprüche unterrepräsentierter Gruppen, etwa von Kindern oder Personen mit seltenen Krankheiten, in die Forschung einbezogen zu werden, haben im Gesetz jedoch keinen Niederschlag gefunden.

\section{Ausblick}

Die vorstehenden Ausführungen haben gezeigt, wie sich seit Mitte des letzten Jahrhunderts auf internationaler Ebene in unterschiedlichen regulatorischen Kontexten und aus unterschiedlichen Motiven ein Bündel von breit akzeptierten Prinzipien zur biomedizinischen Forschung am Menschen herausgebildet hat. Urheber dieser Normen sind vornehmlich internationale Organisationen und Nichtregierungsorganisationen, wobei sich diese häufig gegenseitig wie auch von allenfalls bereits vorhandenen nationalen Forschungsregulierungen inspirieren ließen.

Die internationalen Prinzipien zur Forschung am Menschen wurden von zahlreichen Staaten wie auch der Europäischen Union rezipiert. Ein prägnantes Beispiel für eine solche Rezeption ist, wie vorne dargelegt, die Neuordnung des schweizerischen Humanforschungsrechts. Ein weiteres aktuelles Beispiel ist die am 16.4.2014 erlassene EU Verordnung über klinische Prüfungen mit Humanarzneimitteln $^{65}$. Die Verordnung, die voraussichtlich auf Anfang 2016 in Kraft treten wird, setzt die allgemein anerkannten internationalen Vorgaben in ähnlicher Weise um wie das schweizerische Humanforschungsrecht, allerdings beschränkt auf den Bereich der klinischen Arzneimittelprüfungen. Die Verordnung regelt in detaillierter Weise die materiellen Anforderungen an die Durchführung klinischer Prüfungen ${ }^{66}$ und macht umfangreiche Vorgaben für das Genehmigungsverfahren vor den Ethikkommissionen der Mitgliedstaaten ${ }^{67}$. Was die Transparenz der Humanforschung betrifft, geht die Verordnung weiter als das schweizerische Recht: Gemäß Art. 37 Abs. 4 hat der Sponsor unabhängig vom Ergebnis der klinischen Prüfung innerhalb eines Jahres nach deren Ende eine Zusammenfassung der Ergebnisse der klinischen Prüfung, einschließlich einer laienverständlichen Version, an die grundsätzlich öffentlich zugängliche EU-Datenbank (EudraCT and EudraVigilance) ${ }^{68} \mathrm{zu}$ übermitteln.

59) Vgl. Art. 50 HFG.

60) Art. 48 Abs. 1 und 2 HFG.

61) Dazu oben, sub I.4.

62) Artt. 19 und $20 \mathrm{KlinV}$.

63) Art. $30 \mathrm{KlinV}$.

64) Art. 11 Abs. 1 HFG.

65) Verordnung (EU) Nr. 536/2014 des Europäischen Parlaments und des Rates über klinische Prüfungen mit Humanarzneimitteln und zur Aufhebung der Richtlinie 2001/20/EG.

66) Artt. $28 \mathrm{ff}$. EU-Verordnung über klinische Prüfungen mit Humanarzneimitteln. Für nicht-direktnützige Forschung mit einwilligungsunfähigen Personen erlaubt die Verordnung den Mitgliedstaaten, strengere Anforderungen vorzusehen (Art. 31 Abs. 2). Vgl. sodann Art. 34 (zusätzliche Maßnahmen der Mitgliedstaaten bezüglich Personen, die einen Pflichtwehrdienst leisten, denen die Freiheit entzogen wurde, die aufgrund einer gerichtlichen Entscheidung nicht an einer klinischen Prüfung teilnehmen dürfen oder die in einem Pflegeheim untergebracht sind).

67) Artt. 4 ff. EU-Verordnung über klinische Prüfungen mit Humanarzneimitteln.

68) Vgl. Art. 81 EU-Verordnung über klinische Prüfungen mit Humanarzneimitteln. 